\title{
Visualization of Protease Activity In Vivo Using an Activatable Photo-Acoustic Imaging Probe Based on CuS Nanoparticles
}

\author{
Kai Yang1,2†, Lei Zhu' ${ }^{2,3 \dagger}$, Liming Nie², Xiaolian Sun², Liang Cheng1, Chenxi Wu², Gang Niu², Xiaoyuan \\ Chen ${ }^{\circledR}$ and Zhuang Liu ${ }^{\bowtie}$ \\ 1. Institute of Functional Nano \& Soft Materials (FUNSOM), Soochow University, Suzhou, Jiangsu 215123, China \\ 2. Laboratory of Molecular Imaging and Nanomedicine (LOMIN), National Institute of Biomedical Imaging and Bioengineering (NIBIB), \\ National Institutes of Health (NIH), Bethesda, Maryland 20892, United States \\ 3. Center for Molecular Imaging and Translational Medicine School of Public Health, Xiamen University, Xiamen 361005, China
}

† These authors contributed equally to this work

$\triangle$ Corresponding authors: shawn.chen@nih.gov (X. Chen) or zliu@suda.edu.cn (Zhuang Liu).

() Ivyspring International Publisher. This is an open-access article distributed under the terms of the Creative Commons License (http://creativecommons.org/ licenses/by-nc-nd/3.0/). Reproduction is permitted for personal, noncommercial use, provided that the article is in whole, unmodified, and properly cited.

Received: 2013.07.20; Accepted: 2013.09.14; Published: 2014.01.02

\begin{abstract}
Herein, we for the first time report a novel activatable photoacoustic (PA) imaging nano-probe for in vivo detection of cancer-related matrix metalloproteinases (MMPs). A black hole quencher 3 (BHQ3) which absorbs red light is conjugated to near-infrared (NIR)-absorbing copper sulfide (CuS) nanoparticles via a MMP-cleavable peptide linker. The obtained CuS-peptide-BHQ3 (CPQ) nano-probe exhibits two distinctive absorption peaks at $630 \mathrm{~nm}$ and $930 \mathrm{~nm}$. Inside the tumor microenviorment where MMPs present, the MMP-sensitive peptide would be cleaved, releasing $\mathrm{BHQ} 3$ from the CuS nanoparticles, the former of which as a small molecule is then rapidly cleared out from the tumor, whereas the latter of which as large nanoparticles would retain inside the tumor for a much longer period of time. As the result, the PA signal at $680 \mathrm{~nm}$ which is contributed by BHQ3 would be quickly diminished while that at $930 \mathrm{~nm}$ would be largely retained. The PA signal ratio of $680 \mathrm{~nm} / 930 \mathrm{~nm}$ could thus serve as an in vivo indicator of MMPs activity inside the tumor. Our work presents a novel strategy of in vivo sensing of MMPs based on PA imaging, which should offer remarkably improved detection depth compared with traditional optical imaging techniques.
\end{abstract}

Key words: Peptide, Photoacoustic imaging, Enzyme cleavage, Copper sulfide, MMPs detection.

\section{Introduction}

Matrix metalloproteinases (MMPs) are a family of zinc-dependent endopeptides that degrade proteins in the extracellular matrix (ECM) and play an important role in the development of various diseases including cancer, inflammatory, neurological and cardiovascular diseases [1, 2]. As for cancer, MMPs not only have distinct roles in tumor angiogenesis, but also affect multiple signaling pathways to control the balance between growth and antigrowth signals in the tumor microenvironment [3]. Developing a noninvasive imaging tool to assess MMP activity in vivo would help to understand the roles of MMPs in tumor microenvironments. Recently, various proteases activatable optical probes have been developed for in vivo MMP imaging. These probes usually consist a near-infrared fluorescence dye and a quencher at the opposite ends of an MMP substrate (peptide), and are in their dark quenched state in the absence of MMPs 
[4-6]. Upon exposure to MMPs, the activatable peptide is cleaved by MMPs, releasing the free fluorescent dye which provides the recovered fluorescence to be detected [7-11]. According to the literatures, many groups have reported that optical imaging, especially fluorescence imaging, can be used to detect the MMP activities in vivo $[6,7,12]$. However, the optical imaging has some limitation such as tissue autofluorescence, limited light penetration depth and poor spatial resolution, which may affect the precise of the results.

Photoacoustic (PA) imaging detects ultrasound waves generated by photothermal expansion of light-absorbing tissues or contrast probes under pulsed laser irradiation. As a recently developed imaging technology, PA imaging offers significantly improved in vivo imaging depth and resolution limit compared with traditional in vivo optical imaging [13-15]. While traditional fluorescence imaging usually can only detect signals no deeper than a few millimeters, PA imaging exhibits dramatically enhanced tissue penetration (up to about 7 centimeters so far) and is thus a more clinically relevant imaging technique. Moreover, without the tissue-scattering issue during signal collection, which largely limits the spatial resolution of in vivo fluorescence imaging (e.g. scattering of emitting light by biological tissues), in vivo PA imaging is able offer much better imaging resolution. Nowadays, various organic or inorganic nanoparticles, including porphyrin shell [16], gold nanoparticles [17, 18], carbon nanotubes [19-21] and nano-graphene [22, 23], all with high near-infrared (NIR) absorbance, have widely explored for in vivo PA imaging. However, the use of photoacoustic imaging for detection of the in vivo MMPs activity has not yet been reported to our best knowledge.

In this work, copper sulfide (CuS) nanoparticles, which exhibit strong NIR absorbance and have been widely used as a photothermal agent for cancer ablation therapy [24-26], are conjugated with a red-light-absorbing organic dye, BHQ3, via a MMPs cleavable peptide. The obtained CuS-peptide-BHQ3 (CPQ) nano-probe exhibits strong photoacoustic signal at the wavelengths of $680 \mathrm{~nm}$ and $930 \mathrm{~nm}$ before enzyme cleavage, owing to the absorption of BHQ3 and $\mathrm{CuS}$ nanoparticles, respectively. In the presence of MMPs expressed inside the tumor, our CPQ nano-probe is recognized by the protease, releasing free BHQ3 which as a small molecule could be rapidly washed out from the tumor area, leaving bare CuS nanoparticles which would retain inside the tumor for a much longer period of time (Fig. 1). The PA signal ratios recorded at two different wavelengths, $680 \mathrm{~nm}$ / $930 \mathrm{~nm}$, is then significantly decreased. Therefore, this novel nano-probe allows us for the first time using PA imaging to investigate the in vivo MMP activity in the animal tumor model.

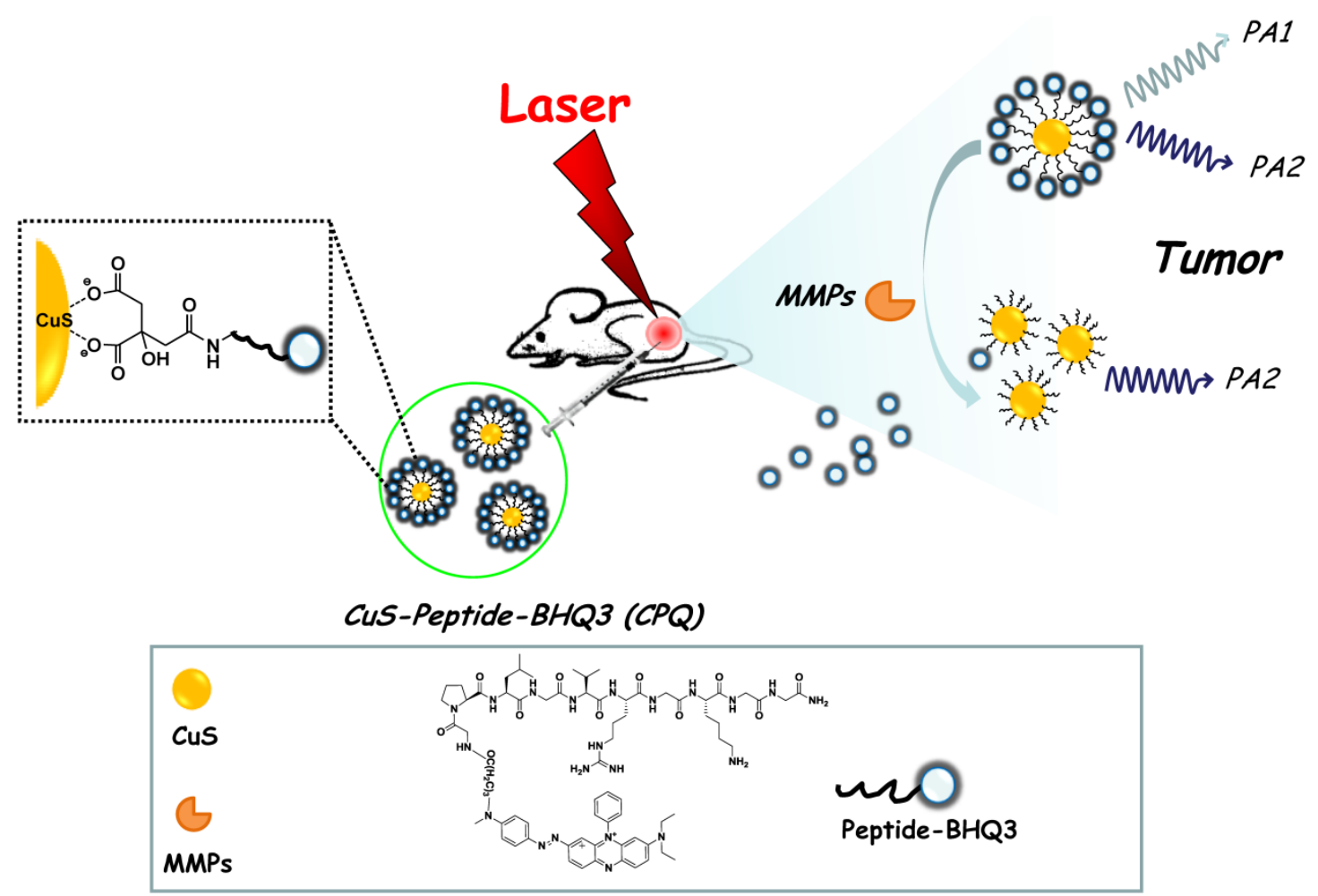

Figure I. A schematic illustration of the CPQ nano-probe that can be activated by MMPs. In this nano-probe, BHQ3 is conjugated to CuS nanoparticles through a MMP-cleavable peptide linker, and thus could be released from nanoparticles upon exposure to MMPs. 


\section{Experimental Section}

\section{Synthesis of copper sulfide nanoparticles}

Copper sulfide (CuS) nanoparticles were synthesized in the aqueous phase. In brief, $14 \mathrm{mg}$ of copper (II) chloride $\left(\mathrm{CuCl}_{2}\right)$ and $20 \mathrm{mg}$ of sodium citrate were dissolved in $100 \mathrm{ml}$ of ultra pure water upon stirring at room temperature. After $5 \mathrm{~min}, 7.8 \mathrm{mg}$ of sodium sulfide was added into the solution. The mixture was reacted at room temperature over night. The citrate-coated CuS nanoparticles were obtained and washed with water by centrifugation at 15,000 $\mathrm{rpm}$ to remove excess reagents. The product was stored in $4^{\circ} \mathrm{C}$ for next experiments.

\section{Synthesis of peptide-BHQ3 probe}

MMP-sensitive substrate peptide was synthesized as previously reported. Briefly, the side chain protected MMP substrate, GPLGVRGKGG, was synthesized manually on 2-Cl-Trt resin at $0.1 \mathrm{mmol}$ scale using the standard Fmoc (N-(9-fluorenyl) methoxycarbonyl) protocol and (benzotriazol-1-yloxy) tripyrrolidinophosphonium hexafluorophosphate (PyBOP) activation. The MMP substrate is recognized and cleaved between Gly and Val as indicated by bold italics. After being released from the resin by Dichloromethane (DCM) containing 1\% trifluoroacetic acid (TFA), the peptide was purified by RP-HPLC on a C18 semi-preparative column using a linear gradient of $10 \%$ to $55 \%$ acetonitrile/water $(0.1 \%$ TFA) for $30 \mathrm{~min}$ at a $10 \mathrm{ml} / \mathrm{min}$ flow rate, and then lyophilized. Next, the side-chain protected MMP substrate was reacted with a black hole quencher-3 (BHQ-3) in DMF solution containing $2 \%$ of DIPEA for 2 hours. After purification, BHQ-3 conjugated MMP substrate (peptide-BHQ3, PQ) was precipitated in cold ether. The pellet was collected by centrifuge and dissolved in TFA cocktail for 2 hours to remove the side chain protection group. After that, free amine on Lysine was exposed for conjugation with CuS. All the final products were confirmed by analytical reversed-phase high performance liquid chromatography (RP-HPLC, DIONEX, CA), using 5\% to $65 \%$ acetonitrile containing $0.1 \%$ TFA versus distilled water containing $0.1 \%$ TFA over $30 \mathrm{~min}$ at a flow rate of $1 \mathrm{ml} / \mathrm{min}$ (C18 column, $5 \mu \mathrm{m}, 120 \mathrm{Ao}, 250 \times 4.6 \mathrm{~mm}$ ) and LC-mass spectroscopy. The purity of the product is $>95 \%$. The obtained peptide-BHQ3 (PQ) probe was dissolved in dimethyl sulphoxide (DMSO) solution and stored in $-20 \mathrm{oC}$

\section{Preparation of CuS-peptide-BHQ3 (CPQ) complex}

$\sim 1 \mathrm{mg}$ of N-(3-dimethylaminopro-pylN'-ethylcarbodiimide) hydrochloride (EDC, Sigma-Aldrich) and $\sim 1 \mathrm{mg}$ of Sulfo-NHS was firstly added to the solution of CuS solution $(0.8 \mathrm{mg} / \mathrm{ml}, 200 \mu \mathrm{l})$ at $\mathrm{pH} 7.4$ to activate the carboxyl group of CuS nanoparticles. After $20 \mathrm{~min}, 10 \mathrm{ml}$ of $\mathrm{PQ}(20 \mathrm{mg} / \mathrm{ml})$ with amino groups was added into the solution and reacted over night at room temperature by avoiding light. Excess PQ was removed by centrifugation at 15,000 rpm and washed away with ultra pure water for two times.

\section{Enzyme activity test}

The activity of the CPQ nano-probe was investigated by incubating the probe $(0.8 \mathrm{mg} / \mathrm{ml})$ in the reaction buffer $(50 \mathrm{mM}$ Tris $\bullet \mathrm{HCl}, 10 \mathrm{mM}$ $\mathrm{CaCl} \cdot 2 \mathrm{H} 2 \mathrm{O}, 0.15 \mathrm{M} \mathrm{NaCl}, 0.05 \%$ Brij35; pH 7.8 TCNB buffer) containing $40 \mathrm{nM}$ of catalytic domain of MMP-13. The UV-vis-NIR spectrums of the samples were measured using a spectrofluorometer (F-7000 Fluorescence Spectrophotometer, Hitach, Tokyo, Japan) at indicated time at $37^{\circ} \mathrm{C}$ for $6 \mathrm{~h}$. Each time before samples were measured, cleaved BHQ3 containing fragment was removed by centrifuge. Three independent experiments were conducted in parallel.

\section{Xenograft tumor models}

All cell culture related reagents were purchased from Gibico. SCC7 breast cancer cells were cultured in RPMI-1640 cell medium supplemented with 10\% FBS and $1 \%$ penicillin-streptomycin. Athymic nude mice were obtained from Harlan laboratories (Frederick, USA) under protocols approved by National Institutes of Health Clinical Center Animal Care and Use Committee (CC/ACUCC). The SCC7 tumor models were generated by subcutaneous injection of $1 \times 10^{6}$ cells in $60 \mu \mathrm{l}$ PBS into the right backside of nude mice. The mice were used for treatment when the tumor volume reached $\sim 100 \mathrm{~mm}^{3}$.

\section{In vivo photoacoustic (PA) imaging}

For PA imaging, nude mice bearing SCC7 tumors were intratumorally injected with CPQ $(100 \mu \mathrm{l}$, $0.8 \mathrm{mg} / \mathrm{ml}$ ) and imaged at different time points post injection by a Vevo 2100 LAZR system (VisualSonics Inc.) at wavelength at $680 \mathrm{~nm}$ and $930 \mathrm{~nm}$ which are contributed by BHQ3 and CuS nanoparticles, respectively. For quantitative calculation, the regions of interest (ROI) were drawn over tumor, with the averaged PA signals measured.

\section{Results}

\section{Preparation of CuS-peptide-BHQ3 (CPQ)}

We prepared CuS nanoparticles in the aqueous solution following a literature protocol [25]. In brief, copper chloride $\left(\mathrm{CuCl}_{2}\right)$ was reacted with sodium sulfide $\left(\mathrm{Na}_{2} \mathrm{~S}\right)$ in the present of sodium citrate upon stirring overnight. The citrate-coated CuS nanoparti- 
cles were obtained and washed by centrifugation at 15,000 rpm to remove excess reagents [25]. Transmission electron microscopy (TEM) image showed that the size of CuS nanoparticles was about $20 \mathrm{~nm}$ (Fig. 2a). UV-vis-NIR spectrum of CuS nanoparticles revealed a high NIR absorbance band (Fig. $2 b$ ). The citrate-coated CuS nanoparticles were very stable in different physiological solutions including phosphate buffer (PBS), fetal bovine serum (FBS) and cell medium (Fig. 2b). In order to study the cytotoxicity of $\mathrm{CuS}$, the standard methyl thiazolyltetrazolium (MTT) assay was carried out to determine relative viabilities of SCC7 breast cancer cells after being incubated with various concentrations of CuS nanoparticles for $24 \mathrm{~h}$. No obvious toxicity of those nanoparticles to cells was observed even at high concentrations up to $100 \mu \mathrm{g} / \mathrm{ml}$ (Supplementary Material: Figure S1).

The enzyme sensitive probe, peptide-BHQ3 (PQ) (BHQ3-Gly-Pro-Leu-GLy-Val-Arg-Gly-Lys-Gly-Gly-( amide)) was synthesized, purified by high performance liquid chromatography (HPLC), and charac- terized by LC-mass spectroscopy, as previously reported[4-6] (Supplementary Material: Figure S2). In order to synthesize the MMP-activatable PA imaging nano-probe, the carboxyl groups on the citrate-coated CuS nanoparticles $(0.8 \mathrm{mg} / \mathrm{ml}, 200 \mu \mathrm{l})$ were firstly activated by N-(3-dimethylaminopropyl $\mathrm{N}$-ethylcarbodiimide) hydrochloride (EDC) and $\mathrm{N}$-Hydroxysulfosuccinimide (Sulfo-NHS). After 20 min, PQ (10 ml, $20 \mathrm{mg} / \mathrm{ml})$ was added into the CuS solution and reacted overnight avoiding light. The obtained product was purified by centrifugation at $15,000 \mathrm{rpm}$ and washed with ultra pure water twice. UV-VIS-NIR spectra uncovered a strong absorption peak at $630 \mathrm{~nm}$, which was attributed to the attachment of BHQ3 on CuS nanoparticles (Fig. 2c). Control experiments evidenced that the PQ was covalently conjugated to CuS nanoparticles instead of physical adsorption (Supplementary Material: Figure S3). A loading efficiency at $12.5 \%$ (PQ: CuS weight ratio) was achieved in this system.

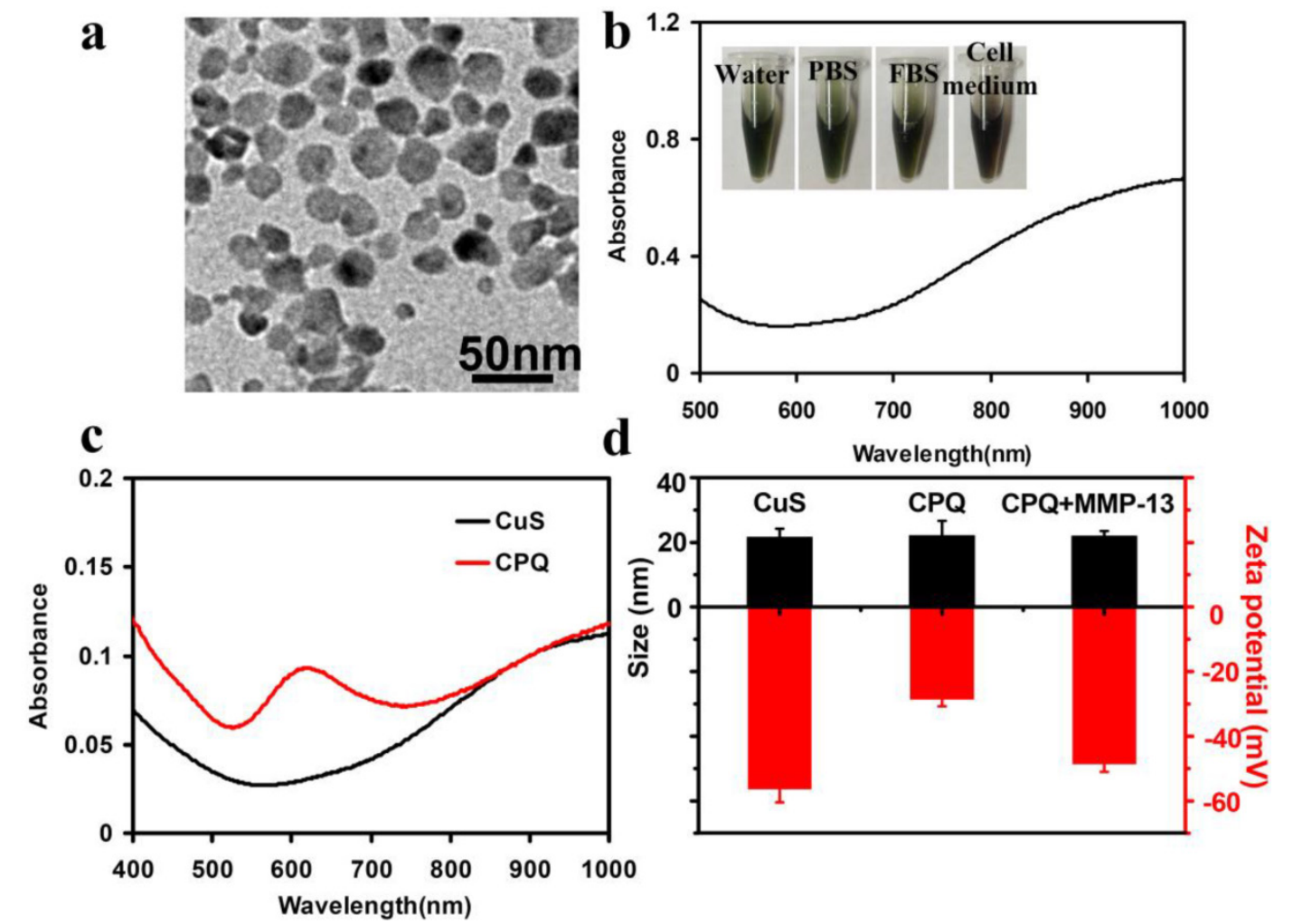

Figure 2. The characterization of CuS nanoparticles and CPQ nano-probe. (a) A TEM image of CuS nanoparticles showing the average particle size at $\sim 20 \mathrm{~nm}$. (b) UV-vis-NIR spectral of CuS nanoparticles in water solution. Inset: the photos of CuS solution $(0.8 \mathrm{mg} / \mathrm{ml})$ in different solutions including water, PBS, FBS and cell medium. (c) UV-vis-NIR spectral of CuS nanoparticles before and after conjugation of peptide-BHQ3. (d)The size and Zeta potential distribution of CuS nanoparticles, CPQ nano-probe, and the CPQ after enzyme cleavage. Conjugation of peptide-BHQ3 resulted in the significant increase of the nanoparticle zeta potential, which decreased again after enzyme cleavage. 

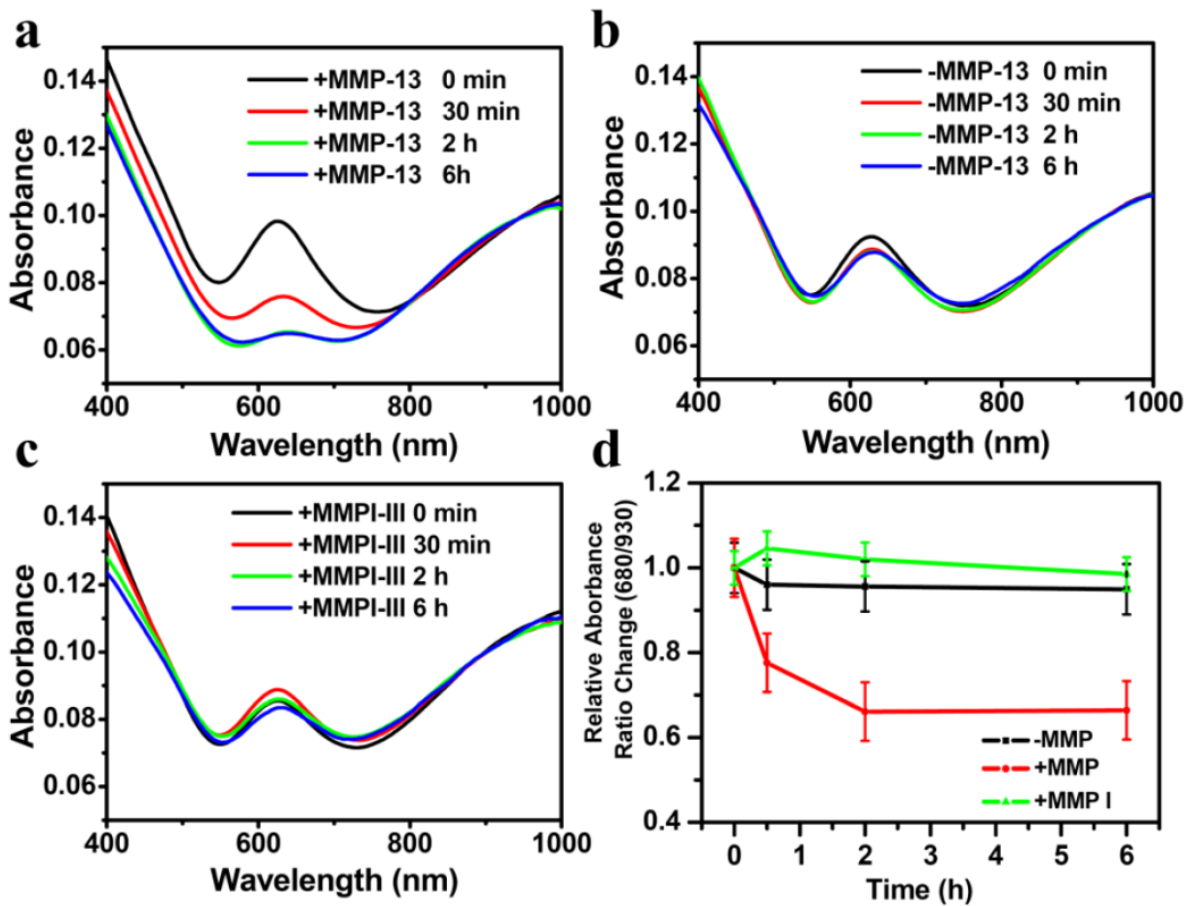

Figure 3. UV-vis-NIR spectra of CPQ nano-probe before and after enzyme cleavage. (a\&b) UV-vis-NIR absorbance spectra of CPQ with (a) or without (b) MMP-I3 addition measured at different time points. (c) UV-vis-NIR absorbance spectra of CPQ added with both MMP-I3 and MMPI-III, the latter of which could inhibit the MMP-activity. (d) The relative absorbance ratio change of 630 and $930 \mathrm{~nm}$ in (a-c). We normalized the relative absorbance ratio $(630 \mathrm{~nm}$ : $930 \mathrm{~nm})$ at the beginning of our experiment to be I. Error bars were based on standard deviations of three parallel samples.

\section{In vitro test the enzyme activity}

In order to demonstrate the response of our nano-probe to MMPs, CPQ $(0.8 \mathrm{mg} / \mathrm{ml}, 100 \mu \mathrm{l})$ was incubated with recombinant human MMP-13 enzyme $(100 \mu \mathrm{l}, 40 \mathrm{nM})$ in the absence or presence of the MMP inhibitor III (MMPI-III, $10 \mu \mathrm{l}, 50 \mathrm{nM}$ ), a broad spectrum MMP inhibitor that could inhibit the activities of many MMPs including MMP- 13 , at $37^{\circ} \mathrm{C}$ in the TCNB buffer solution $(50 \mathrm{mM}$ Tris $\bullet \mathrm{HCl}, 10 \mathrm{mM}$ $\mathrm{CaCl}_{2} \cdot 2 \mathrm{H}_{2} \mathrm{O}, 0.15 \mathrm{M} \mathrm{NaCl}, 0.05 \%$ Brij35; pH 7.8). At different time intervals, portions of the solutions were collected and centrifuged at 15,000 rpm for $5 \mathrm{~min}$ to remove supernatant. The sediment was then re-dissolved in water and recorded by UV-vis-NIR spectrometer. An obvious decrease of the absorption peak at $630 \mathrm{~nm}$ was observed for CPQ added with MMP-13 within 2 hours, owing to the protease induced peptide cleavage that released free BHQ3 (Fig 3a). In marked contrast, no significant change in the UV-VIS-NIR spectra of CPQ without adding MMP-13, or the mixture of $\mathrm{CPQ}$ and MMP-13 but with addition of protease inhibitor MMPI-III, was noticed, suggesting our CPQ nano-probe was stable in the absence of MMP-13 activity (Fig 3b\&c). Based on the change of absorbance ratio at $630 \mathrm{~nm}$, we can then use this nano-probe to determine MMPs activity (Fig. 3d).

\section{In vivo MMP activity test}

Before using photoacoustic imaging to detect the activity of MMPs in vivo, we first measure the PA signals of free BHQ3 $(0.1 \mathrm{mg} / \mathrm{ml})$, free CuS nanoparticles $(0.8 \mathrm{mg} / \mathrm{ml})$, and CPQ $(0.8 \mathrm{mg} / \mathrm{ml})$ before and after enzyme cleavage in solutions. Because the wavelength range of the laser in our PA instrument was $680-970 \mathrm{~nm}$, we chose $680 \mathrm{~nm}$ instead of the peaked absorbance wavelength $(630 \mathrm{~nm})$ for BHQ3 imaging. While the PA signals of free $\mathrm{BHQ} 3$ were rather strong at $680 \mathrm{~nm}$ but barely detectable at 930 $\mathrm{nm}, \mathrm{CuS}$ nanoparticles offered much stronger PA signals at $930 \mathrm{~nm}$ than that at $680 \mathrm{~nm}$ (Fig 4a). As expected, CPQ nano-probe showed strong PA signals at both $680 \mathrm{~nm}$ and $930 \mathrm{~nm}$ prior to enzyme cleavage (Fig. 4a). However, after being treated with MMP-13 and purification to remove detached dye molecules, the PA signal of CPQ at $680 \mathrm{~nm}$ was significantly decreased, while that at $930 \mathrm{~nm}$ had little change (Fig. 4a), consistent to the absorbance data (Fig. 2a).

Nanoparticles with large sizes usually would show much longer retention in the tumor compared to small molecules, which are easily washed out and excreted after administration. This is the basic assumption of our MMP-responsive PA imaging nano-probe. To verify our speculation, mice bearing SCC7 tumors were intratumorally injected with free BHQ3 $(0.1 \mathrm{mg} / \mathrm{ml}, 100 \mu \mathrm{l})$ and free $\mathrm{CuS}$ nanoparticles $(0.8 \mathrm{mg} / \mathrm{ml}, 100 \mu \mathrm{l})$, and imaged by a Vevo $2100 \mathrm{LAZR}$ system (Visual Sonics Inc.). Right after injection, BHQ3 treated mice exhibited very strong $680 \mathrm{~nm}$ PA 
signals, which was rapidly diminished in just 2 hours (Fig 4 b\&c). In contrast, CuS nanoparticles injected tumors exhibited high $930 \mathrm{~nm}$ PA signals, which retained almost at constant level over 24 hours (Fig 4 $b \& c)$.

At last, we used our CPQ nano-probe for in vivo MMPs detection by PA imaging. The SCC7 tumor modal, which has been extensively documented to show high levels of MMP-13 expression [4-7, 12, 27], was chosen in our experiments. After intratumoral injection of CPQ $(0.8 \mathrm{mg} / \mathrm{ml})$, PA images were taken using $680 \mathrm{~nm}$ and $930 \mathrm{~nm}$ excitations. Right after injection, strong PA signals at both $680 \mathrm{~nm}$ and $930 \mathrm{~nm}$ showed up in the SCC7 tumor. Interestingly, the PA signals at $680 \mathrm{~nm}$ remarkably decreased over time due to MMP-induced peptide cleavage that released BHQ3 (Fig 4b), while that at $930 \mathrm{~nm}$ showed little change within $24 \mathrm{~h}$ (Fig $4 \mathrm{~b} \& \mathrm{~d})$. Note that since the MMP-induced cleavage of the CPQ probe as well as the washing out of released BHQ3 molecules from the tumor all took time, the PA signals at $680 \mathrm{~nm}$ showed no significant changes in the first 2 hours but dramatically decreased at later time points. In order to confirm this phenomenon was due to MMPs induced specific cleavage, MMP inhibitor-III was injected into the tumor $30 \mathrm{~min}$ prior to the administration of CPQ nano-probe to inhibit MMP-activity. In this case, no significant change of PA signals at both $680 \mathrm{~nm}$ and $930 \mathrm{~nm}$ was observed within 24 hours post injection, suggesting that the MMP inhibitor could inhibit the MMPs enzyme activity in vivo and prevent enzyme-induced cleavage of the MMP-specific peptide (Fig 4b\&d).

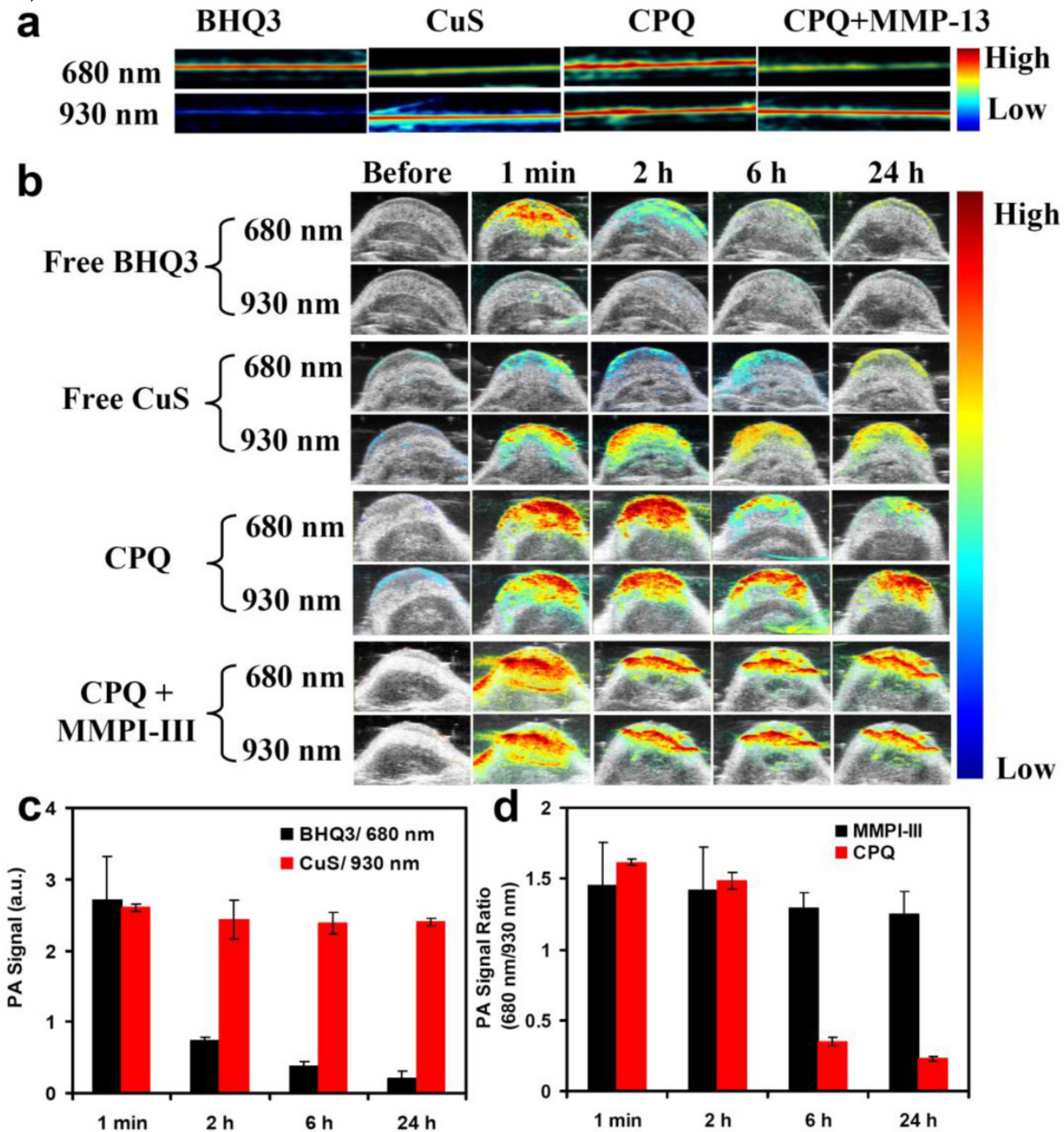

Figure 4. In vivo MMP-sensing by photocoustic imaging. (a) Solution PA images of BHQ3 (0.l mg/ml), CuS (0.8 mg/ml), CPQ nano-probe (0.8 mg/ml), and CPQ + MMP-13. (b) In vivo PA imaging of mice with tumors injected with free BHQ3, free CuS, CPQ nano-probe, and CPQ with MMPI-III co-injection. The images were taken at different time post injection. (c) The change of tumor PA signals of the control groups including free BHQ3 at $680 \mathrm{~nm}$ and free CuS nanoparticles at $930 \mathrm{~nm}$. (d) The PA signal ratios $(680 \mathrm{~nm} / 930 \mathrm{~nm})$ of the CPQ group and CPQ + MMPI-III group measured at different time post injection. The background PA signal before injection was subtracted in the above values. Error bars were based on standard deviations of 3-4 mice per group. The $680 \mathrm{~nm} / 930 \mathrm{~nm}$ PA signal ratio at the tumor after injection of CPQ nano-probe could be used to detect MMP-activities in vivo. 
In order to accurately calculate the PA signal at $680 \mathrm{~nm}$ and $930 \mathrm{~nm}$, we drew up the region-of-interest (ROI) in the PA images to count the PA signals, with the background PA signals (before injection of CPQ nano-probe) subtracted. The PA signal ratio of 680 $\mathrm{nm}: 930 \mathrm{~nm}$ was calculated. It was found that in tumors injected with the CPQ probe, this ratio showed a remarkable decrease, which was not observed if MMPI-III was pre-injected to inhibit the MMP-activity inside the tumor (Fig. 4d). Therefore, we could use this nano-probe to detect the in vivo MMP-activity by PA imaging.

\section{Conclusion}

We have demonstrated a MMP enzyme-sensitive nano-probe with $\mathrm{CuS}$ nanoparticles and BHQ3 molecules conjugated together though a MMP-cleavable peptide linker for in vivo MMP detection under PA imaging. While showing strong PA signals at both 680 $\mathrm{nm}$ and $930 \mathrm{~nm}$ wavelengths owing to the optical absorbance of BHQ3 and CuS nanoparticles, respectively, the CPQ nano-probe in the presence of MMPs can be tailored to discard $\mathrm{BHQ3}$, resulting in remarkably decreased PA signals at $680 \mathrm{~nm}$ in comparison to that at $930 \mathrm{~nm}$. Such a simple and straightforward strategy allows us for the first time to use PA imaging for in vivo detection of a specific enzyme activity. Compared with traditional optical imaging (e.g. fluorescence imaging) used in MMP detection, PA imaging would exhibit dramatically enhanced tissue penetration, and is expected to offer markedly improved in vivo spatial resolution during imaging owing to its unique mechanism[15, 17]. Our results would encourage further exploration of smart imaging probes for in vivo detection of other classes of important functional biological molecules based on PA imaging.

\section{Supplementary Material}

Fig.S1 - Fig.S3.

http://www.thno.org/v04p0134s1.pdf

\section{Acknowledgements}

This work was partially supported by the National "973" Program of China (2011CB911002, 2012CB932601, 2013CB733802, 2014CB744503), the National Natural Science Foundation of China (51002100, 51072126, 51373144, 81371596), and the Intramural Research Program (IRP) of the National Institute of Biomedical Imaging and Bioengineering (NIBIB), NCI), National Institutes of Health (NIH). Kai Yang was supported by a scholarship from the China scholarship Council (CSC).

\section{Competing Interests}

The authors have declared that no competing interest exists.

\section{References}

1. Liotta LA, Tryggvason K, Garbisa S, Hart I, Foltz CM, Shafie S. Metastatic Potential Correlates With Enzymatic Degradation Of Basement-Membrane Collagen. Nature. 1980; 284: 67-8.

2. Egeblad M, Werb Z. New functions for the matrix metalloproteinases in cancer progression. Nature Reviews Cancer. 2002; 2: 161-74.

3. Kessenbrock K, Plaks V, Werb Z. Matrix Metalloproteinases: Regulators of the Tumor Microenvironment. Cell. 2010; 141: 52-67.

4. Zhu L, Xie J, Swierczewska M, Zhang F, Quan Q, Ma Y, et al. Real-Time Video Imaging of Protease Expression In Vivo. Theranostics. 2011; 1: 18-27.

5. Zhu L, Wang H, Wang L, Wang Y, Jiang K, Li C, et al. High-affinity peptide against MT1-MMP for in vivo tumor imaging. Journal Of Controlled Release. 2011; 150: 248-55.

6. Zhu L, Zhang F, Ma Y, Liu G, Kim K, Fang X, et al. In Vivo Optical Imaging of Membrane-Type Matrix Metalloproteinase (MT-MMP) Activity. Molecular Pharmaceutics. 2011; 8: 2331-8.

7. Lee S, Cha E-J, Park K, Lee S-Y, Hong J-K, Sun I-C, et al. A near-infrared-fluorescence-quenched gold-nanoparticle imaging probe for in vivo drug screening and protease activity determination. Angewandte Chemie-International Edition. 2008; 47: 2804-7.

8. Lu W, Melancon MP, Xiong C, Huang Q, Elliott A, Song S, et al. Effects of Photoacoustic Imaging and Photothermal Ablation Therapy Mediated by Targeted Hollow Gold Nanospheres in an Orthotopic Mouse Xenograft Model of Glioma. Cancer Research. 2011; 71: 6116-21.

9. Xia X, Yang M, Oetjen LK, Zhang Y, Li O, Chen J, et al. An enzyme-sensitive probe for photoacoustic imaging and fluorescence detection of protease activity. Nanoscale. 2012; 3: 950-3

10. Yao H, Zhang Y, Xiao F, Xia Z, Rao J. Quantum dot/bioluminescence resonance energy transfer based highly sensitive detection of proteases. Angewandte Chemie-International Edition. 2007; 46: 4346-9.

11. Xia Z, Xing Y, So M-K, Koh AL, Sinclair R, Rao J. Multiplex Detection of Protease Activity with Quantum Dot Nanosensors Prepared by Intein-Mediated Specific Bioconjugation. Analytical Chemistry. 2008; 80: 8649-55.

12. Lee S, Ryu JH, Park K, Lee A, Lee S-Y, Youn I-C, et al. Polymeric Nanoparticle-Based Activatable Near-Infrared Nanosensor for Protease Determination In Vivo. Nano Letters. 2009; 9: 4412-6.

13. De La Zerda A, Zavaleta C, Keren S, Vaithilingam S, Bodapati S, Liu Z, et al. Carbon nanotubes as photoacoustic molecular imaging agents in living mice. Nature Nanotechnology. 2008; 3: 557-62.

14. Wang LV. Multiscale photoacoustic microscopy and computed tomography. Nature Photonics. 2009; 3: 503-9.

15. Wang LV, Hu S. Photoacoustic Tomography: In Vivo Imaging from Organelles to Organs. Science. 2012; 335: 1458-62.

16. Huynh E, Lovell JF, Helfield BL, Jeon M, Kim C, Goertz DE, et al. Porphyrin Shell Microbubbles with Intrinsic Ultrasound and Photoacoustic Properties. Journal Of The American Chemical Society. 2012; 134: 16464-7.

17. Kim J-W, Galanzha EI, Shashkov EV, Moon H-M, Zharov VP. Golden carbon nanotubes as multimodal photoacoustic and photothermal high-contrast molecular agents. Nature Nanotechnology. 2009; 4: 688-94.

18. Kircher MF, de la Zerda A, Jokerst JV, Zavaleta CL, Kempen PJ, Mittra E, et al. A brain tumor molecular imaging strategy using a new triple-modality MRI-photoacoustic-Raman nanoparticle. Nature Medicine. 2012; 18: 829-U235.

19. de la Zerda A, Liu ZA, Bodapati S, Teed R, Vaithilingam S, Khuri-Yakub BT, et al. Ultrahigh Sensitivity Carbon Nanotube Agents for Photoacoustic Molecular Imaging in Living Mice. Nano Letters. 2010; 10: 2168-72.

20. de la Zerda A, Bodapati S, Teed R, May SY, Tabakman SM, Liu Z, et al. Family of enhanced photoacoustic imaging agents for high-sensitivity and multiplexing studies in living mice. ACS nano. 2012; 6: 4694-701.

21. Wang C, Ma X, Ye S, Cheng L, Yang K, Guo L, et al. Protamine Functionalized Single-Walled Carbon Nanotubes for Stem Cell Labeling and In Vivo Raman/Magnetic Resonance/Photoacoustic Triple-Modal Imaging. Advanced Functional Materials. 2012; 22: 2363-75.

22. Yang K, Feng L, Shi X, Liu Z. Nano-graphene in biomedicine: theranostic applications. Chemical Society Reviews. 2013; 42: 530-47.

23. Yang K, Hu L, Ma X, Ye S, Cheng L, Shi X, et al. Multimodal Imaging Guided Photothermal Therapy using Functionalized Graphene Nanosheets Anchored with Magnetic Nanoparticles. Advanced Materials. 2012; 24: 1868-72.

24. Tian Q, Jiang F, Zou R, Liu O, Chen Z, Zhu M, et al. Hydrophilic Cu9S5 Nanocrystals: A Photothermal Agent with a 25.7\% Heat Conversion Efficiency for Photothermal Ablation of Cancer Cells in Vivo. Acs Nano. 2011; 5: 9761-71.

25. Zhou M, Zhang R, Huang M, Lu W, Song S, Melancon MP, et al. A Chelator-Free Multifunctional [Cu-64]CuS Nanoparticle Platform for Simultaneous Micro-PET/CT Imaging and Photothermal Ablation Therapy. Journal Of The American Chemical Society. 2010; 132: 15351-8. 
26. Ku G, Zhou M, Song S, Huang Q, Hazle J, Li C. Copper Sulfide Nanoparticles As a New Class of Photoacoustic Contrast Agent for Deep Tissue Imaging at $1064 \mathrm{~nm}$. Acs Nano. 2012; 6: 7489-96.

27. Ala-aho R, Ahonen M, George SJ, Heikkila J, Grenman R, Kallajoki M, et al. Targeted inhibition of human collagenase-3 (MMP-13) expression inhibits squamous cell carcinoma growth in vivo. Oncogene. 2004; 23: 5111-23. 